\title{
A New Factor for Computing the Relevance of a Document to a Query
}

\author{
Mohand Boughanem, Ihab Mallak, and Henri Prade
}

\begin{abstract}
In this paper we propose a method for semantic text representation and term weighting. It is based on a semantic resource, WordNet, that provides meaning information and relations between the terms of a document. The heart of the proposed method is the way the concepts (terms) of documents are clustered and weighted. More precisely, we introduce two notions: the "centrality" of a term and its specificity. The centrality of a term is given by the number of terms of the document that are directly related to it in the same conceptual cluster. The "specificity" represents the depth of a concept in WordNet. These parameters are different from the usual term frequency "tf" and inverse term frequency "idf" used in classical information retrieval. This method is based on two steps: 1) matching document terms with concepts of "WordNet" in order to obtain the most appropriate ones 2) for each concept calculating its centrality using existing semantic "WordNet" relations, and its "specificity". The preliminary experiments undertaken on TREC collections show the effective interest of these parameters.
\end{abstract}

\section{INTRODUCTION}

Most information retrieval models that compute the relevance of a document to a query, such as vector space [1], probabilistic [2], inference network [3], LM [4] compute a score using term weights in the document and query. These weights are usually based on a combination of parameters like term frequency (tf), inverse document frequency (idf) and document length $\left(1_{d}\right)$.

The aim of this paper is to propose two new parameters for evaluating document relevance w.r.t. a query. These parameters go beyond the simple counting of terms. They attempts to measure 1) how a query term is related (semantically) to other terms of a given document, this factor is called "centrality" and 2) how much a query term is specific or generic in a given context.

These parameters are computed using an "ontology". In fact any resource that provides semantic relationships between terms could be used. More precisely, the "centrality" is estimated for each query term by counting the number of terms of a document that are related to it using existing semantic "WordNet" relations between terms. The "specificity" represents the depth of a term, from the root to it, in "WordNet".

The paper is organized as follows. Section 2 provides a brief review on term weighting and text representation approaches. Section 3 details the new term weighting factors for document ranking, namely centrality and specificity. Section 4 presents the experimental settings and results. The

Mohand Boughanem, Ihab Mallak and Henri Prade are with IRIT at University of Toulouse, 118 route de Narbonne, 31062 Toulouse cedex 09, France (email: \{bougha, mallak, prade\}@irit.fr). experiments were undertaken on TREC collection. We compare our approach to BM25 term weighting [5] and to a concept-based method proposed by Baziz [6]. We then conclude and give some perspective tracks.

\section{RELATED WORKS}

In the following sections, we briefly review some approaches about term weighting, and then some approaches about text representation.

\section{A. Term Weighting Approaches}

Most of existing approaches assign a weight to a term and then compute and compare the relevance of a document w.r.t. to query by matching the query terms with document terms. Therefore, if a document does not contain a query term this document has no chance to be selected. For weighting term, these approaches are based on three factors: the term frequency factor, the inverse document frequency factor, and the document length factor. The final term weight is the product of these three factors. Numerous term weighting schemas have been developed within the tf.idf family [2][29][30][8][9][10]. Among them, the Okapi formula [30] is one of the most popular term weighting methods.

Other approaches automatically learn term weights by incorporating information about the category of documents as proposed by Rong Jin et al. [31]. Such approaches assume that the semantic meaning of a document can be represented, at least partially, by the set of categories to which it belongs.

\section{B. Text Representation}

Textual documents can be represented (indexed) by considering different features which could be either terms (keywords, phrases, concepts) or topics. The majority of the approaches to term extraction from texts are based on the same techniques. They consist in seeing a document as a bag of weighted words [7]. The weights estimate the degree of importance of a word with respect to the description of the document. As already said, several techniques were developed, the most widely spread and used are the ones based on the $t f . i d f$ type of index ( $t f$ reflects the frequency of the term in the document, while $i d f$ accounts for the inverse frequency of the term in a collection) [8][9][10].

Various approaches have been proposed to go beyond the simplistic bag of words approaches. They attempt at identifying and extracting word sense, or concepts occurring in the documents. Two main approaches have been undertaken in information retrieval (IR): the semantic-based approach and the concept-based approach. The first one tries to extract the sense of the terms (single words or phrases), either by analyzing the words in their context [11][12][13], or by using a distribution of pairs of terms in the collection 
of documents, as it is done in latent semantic indexing (LSI) [14].

The second type of approach tries to identify and to extract the concepts appearing in documents. These concepts are often extracted from an external resource (dictionary, thesaurus, ontology). In [15], [16] the use of abstract graphs for representing documents and queries is discussed. The authors propose a method to measure the similarity of sentences represented by abstract graphs. Gonzalo et al. [17] propose an indexing method based on the WordNet synsets. The vector space model is employed, using synsets as indexing space instead of word forms. In a similar spirit, Richardson et al. [18] have proposed to represent the documents and the queries on the basis of concepts names extracted from WordNet. An early example of the use of ontologyes for refining queries is proposed in [19]. Fuzzy similarity interrelation, as well as generality interrelation, based on a fuzzy synonymy dictionary and a fuzzy ontology has been proposed by Olivas et al. [20] for making a readjustment of term weights in documents, and generating new queries. Boughanem et al. [21] have proposed a general conceptual IR model, which represents documents and queries requests as sub-trees of concepts (nodes) issued from an ontology. The document and query representations are not only set of concepts occurring in their contents, but they are completed by intermediate concepts (nodes).

\section{The Idea of Centrality}

Some idea of centrality is naturally related to the notion of cluster. For instance, in graph clustering, a notion of topological centrality reflecting the topological positions of nodes and edges in general networks is currently used; see, e.g. [34]. The idea of centrality can be encountered in different domains: i) in web search for developing more accurate topic-classification algorithms where the pages and hyperlinks of the World-Wide Web may be viewed as nodes and edges in a directed graph [36]; ii) in social networks, where centrality is widely used for representing the central actor that is most likely working on central subjects [33]. Another use of an idea of centrality may be found in the computation of degrees of relatedness between semantic entries and their various categories [32]. In text summarization, in order to compute the centrality of a sentence in a document [35], a graph-based method for computing relative importance of textual units for natural language processing has been used. In this method, the centrality of a sentence is defined mainly in terms of the centrality of the words that it contains. A common way of assessing word centrality is to look at the centroid of the document cluster in a vector space. The centroid of a cluster is a pseudo-document that consists of words that have tf*idf scores above a predefined threshold, where tf is the frequency of a word in the cluster, and idf values are typically computed over a much larger but similar data collection.

In this paper we are making a quite different use of the idea of centrality, which is now one of the parameters used for rank-ordering documents that are relevant w.r.t. a query in information retrieval. This is an original feature of the approach presented in this paper, where we compute the centrality of a term in document on the basis of its semantic relations with other terms of the document.

\section{NEW TERM WEIGHTING FACTORS FOR DOCUMENT RANKING}

The idea behind the document ranking approach that we are investigating attempts first to go beyond the usual termterm matching largely used in IR and secondly to use new sources of evidence for term weighting. Two sources of evidence are proposed. The first one attempt to measure how much a query term is related to other terms of a given document. This relationship is called centrality. We assume that the more a query term is related to other document term (higher is its centrality) and the more the document is likely to be relevant for the query. The second factor, the specificity, estimates how much a term is specific or generic (for example the term "vehicle" is a generic term and the term "car" is more specific where "car" refers to a kind of "vehicle"). Then each of the query terms may have different meanings that depend on the studied document, and for each sense we have a degree of specificity. We assume that more the query terms are specific in a studied document, the more the document is likely to be relevant to the query.

In order to measure these two parameters, we need to use a resource that provides relationships between terms. In our experiments we use "WordNet" [23], which groups English words into concepts (sets of ,synonyms ${ }^{e e}$ called, synsetse). In WordNet, there are six main relations that may hold between a pair of words (synonyms, glossary, specializes, a part of, in the same domain, and related to). We should note that each word (synsets) of WordNet has a specificity that is estimated through its depth in the conceptual tree induced by the "is-a" relation in WordNet.

The first step of our approach is to build a conceptual representation of a document and a query. The next sections detail this conceptual representation and then the weighting factors.

\section{A. Concept-Based Representation of Documents and Queries}

In the proposed approach, the query evaluation is mediated by an ontology $O$ of concepts, which are supposed to be sufficient for describing the contents of the considered documents with an appropriate level of accuracy. Leaves in $O$ can be thought as simple keywords, i.e. keywords expressing specialized concepts, while other nodes refer to keywords, which are labels of more general concepts. Edges in this ontology represent the classical is-a link.

Both documents and queries are supposed to be interpreted or expressed in terms of labels of nodes of $O$.

Let $d$ be a document and $q$ a query. Each document $d$ (resp. query $q$ ) is identified by a set of terms $R_{d}=\left\{t_{i} / i=1\right.$, $K(d)\}$ (resp. $R_{q}=\left\{t_{i} / i=1, K(q)\right\}$ ) where $t_{i}$ is a key word or multi-word taken from $d$ (resp. from $q$ ). We only deal with lemmatized noun groups and $K(d)$ (resp. $K(q)$ ) is the number of terms in document $d$ (resp. in query $q$ ).

The first step of our approach is to project $R_{d}$ on $O$ and to identify, for each $t_{i} \in R_{d}$, a unique concept $c_{j}$ in $O$. 
Namely $N(O, d)=\left\{c_{j} / j=1, m(d)\right\}$ where $c_{j}$ are concepts (or node) of ontology, and for any concept $c_{j} \in N(O, d), \exists t_{i} \in R_{d}$ associated to $c_{j \cdot} m(d)$ is the number of concepts in $O$ that are equivalent to some terms in $R_{d}$. When several equivalent expressions $t_{i}$ in $O$ exist, the longest concept is retained. Each noun $t_{i} \in R_{d}$ may belong to many concepts in $O$ according to its representing meaning. To choose the correspondent meaning concept of a noun we use the disambiguation method proposed by [6].

As a result, each document is represented by a bag of disambiguated concepts of the ontology:

$\mathrm{d}=N(O, d)=\left\{c_{j} / j=1, m(d)\right\}$

where $c_{j}$ is a disambiguated ontology concept that corresponds to a noun in the document, and $m(d)$ is the number of identified concepts from document $d$.

The second step of the approach is also to project the terms of the query $R_{q}$ on $O$ and to identify for each $t_{i} \in R_{q}$ the different existing concepts in $O$, namely $N(O, q)=\left\{c_{j} / j=\right.$ $1, m(q)\}$, where $c_{j}$ are concepts (or nodes) of the ontology and for any concept $c_{j} \in N(O, q), \exists t_{i} \in R_{q} . m(q)$ is the number of concepts in $O$ that are equivalent to some terms in $R_{q}$. Note that no disambiguation method is used for the query; then for each noun of $R_{q}$ it exists one or more correspondent concepts $c_{j} \in N(O, q)$.

As result, each query is represented as a bag of concepts of the ontology:

$\mathrm{q}=N(O, q)=\left\{c_{j} / j=1, m(q)\right\}$

where $c_{j}$ is an ontology concept that corresponds to a word in the query, and $m(q)$ is the number of identified concepts in query.

\section{B. "Semantic" Term Weighting}

The heart of the proposed term weighting method is term centrality. We attempt to measure the centrality of a term by considering its relatedness to concepts of the document. The more a concept is related to other concepts of a document, the higher is its centrality. The easiest way to compute the centrality of a given term $t$ is by counting the number of document concepts that are related to it. The relations between concepts are those provided by ontology $O$, in our case "WordNet".

Formally, the Centrality " $C_{t, d}$ " of a concept $t$ in a document $d$, is estimated by the number of concepts of $d$ that are related to $t$.

$C_{t, d}=\left\{\# R\left(t, t_{k}\right) \forall t_{k} \in d\right\}$,

where $\# R\left(t, t_{k}\right)$ is the number of relations between concept $t$ and concepts $t_{k}$.

The second parameter we introduce is Specificity " $S_{t}$ " of a concept $t$ in ontology $O$.

$S_{t}=\# i s-a(t$, root node $)$ is estimated by the number of "is-a" relations that separate concept $t$ from the root-node of the Ontology hierarchy.

The third parameter we use is frequency " $\mathrm{F}_{\mathrm{t}, \mathrm{d}}$ " that is estimated by the number of occurrences of the term $t$ in the document $d$.

The ranking model we propose is based on the following combination of these three parameters:

$$
\operatorname{RSV}(\mathrm{q}, \mathrm{d})=\sum_{t=q}\left(\left(1+C_{t, d}\right)^{\alpha}=\left(S_{t}\right)^{\beta}=\left(1+F_{t, d}\right)^{\gamma}\right)
$$

where $\mathrm{q}$ is a query now composed of concepts, $\mathrm{d}$ a document, $\alpha, \beta$ and $\gamma$ are parameters to be optimized.

In our RSV model we used " $(1+\mathrm{C})$ " and " $(1+\mathrm{F})$ " instead of "C" and "F", because a term of a query may not appear in the document, so its frequency will be zeo $(\mathrm{F}=0)$, or may not have any word that is related to it in the document then its centrality will be zero $(\mathrm{C}=0)$.

\section{EXPERIMENTATION}

Experiments were carried out on TREC1 collection. The documents of TREC1 are issued from Disk1\&2 and composed of newspapers articles. The queries are issued from the topics 51-100. This collection is provided by TREC-NIST. The collection is composed of a set of 741670 documents, a set of topics (queries) and a set of relevance judgments corresponding to the relevant documents per query.

To evaluate our system we use the following this protocol. The documents were first indexed using a classical term indexing. It consists in selecting single words occurring in the documents, and then stemming words using Porter algorithm [25] and at the end removing stop-words according to a standard list [26]. A weight is then assigned to each term following a kind of BM25 TF.IDF formula of Robertson and Walker [5]. The same process is applied to queries. A vector-based model is then used to retrieve documents. More precisely each query is submitted to the Mercure system [24], the system returns the top 1000 documents. These results are considered as the baseline.

In order to evaluate the effectiveness of our proposal, we attempt, in these preliminary experiments, to rerank the top 100 retrieved documents of the baseline by considering the new factors. The effectiveness is evaluated in terms of precision and recall points. The precision at point $\mathrm{x}, \mathrm{Px}$, is the ratio evaluating the number of relevant documents retrieved among the $\mathrm{x}$ top retrieved documents. Moreover, to better evaluate whether the obtained results, mainly when comparing two methods, are statistically significant, we performed a statistical significance test, namely Student paired t-test. This t-test assigns a confidence value ( $p$-value) to the null hypothesis. The typical null hypothesis is that there is no difference between the compared systems. When the p-value is low, typically if p-value $<0.05$, the null hypothesis is rejected (which means that the results are not obtained by chance).

Our aims in these experiments are threefold:

- Evaluate the effect of the new parameters, namely term centrality and term specificity.

- Compare our best results precision to those obtained by the BM25 (Best Matching 25).

- Finally, compare our best result to a conceptual indexation method proposed by Baziz [6].

\section{A. The Proposed Approach}

The aim of this section is to find the impact of each of the above described parameters "centrality, specificity and 
frequency" in order to re-order the 100 documents retrieved for each of the 50 queries obtained by the BM25 (Best Matching 25) of the probabilistic model OKAPI of Robertson and Walker [5]. Next we "ll aggregate these parameters to find the function that gives the best ranking results.

As mentioned above, we used "WordNet" [23] as semantic resource. WordNet is a lexical database for the English language. It groups English words into sets of synonyms called synsets, provides short, general definitions, and records the various semantic relations between these synonym sets. The purpose is twofold: to produce a combination of dictionary and thesaurus that is more intuitively usable, and to support automatic text analysis and artificial intelligence applications.

In WordNet, there are six main relations that may hold between a pair of words (or word expressions) $w$ and $w^{\prime}$. They are

- $\quad-w \mathrm{~S} w^{\prime}: w$ and $w^{\prime}$ are synonyms;

- $\quad-w \mathrm{G} w^{\prime}: w$ is in the glossary definition of $w^{\prime}$;

- $\quad$ - $w$ I $w^{\prime}: w$ specializes $w^{\prime}$ ("is-a" relation); then $w^{\prime} \mathrm{I}^{-1}$ $w$ reads $w^{\prime}$ generalizes $w$

- $\quad-\quad w \mathrm{P} w^{\prime}: w$ is a part of $\mathrm{w}$; conversely $w^{\prime} \mathrm{P}^{-1} w$ reads $w^{\prime}$ is composed of $w$;

- $\quad-w \mathrm{D} w^{\prime}: w$ and $w^{\prime}$ are in the same domain;

- $\quad-w \mathrm{R}$ w': $w$ is related to $w^{\prime}$.

Relations S, D, and R are symmetrical, while G, I and P are not. Besides, S, I and D are transitive. Moreover, it is possible to define new relations from these relations by taking their unions: thus, $w\left(\mathrm{R}^{\mathrm{i}} \cup \mathrm{R}^{\mathrm{j}}\right) w^{\prime}$ would mean that $w$ is in relation $\mathrm{R}^{\mathrm{i}}$ or $\mathrm{R}^{\mathrm{j}}$ with $w^{\prime}$. One may also think of composing relations when they are not transitive: $w \mathrm{R}^{\mathrm{i}} \mathrm{oR}^{\mathrm{i}} w^{\prime}$ if $\exists \mathrm{w}^{\circ}, w \mathrm{R}^{\mathrm{i}} w^{\circ}$ and $w^{\circ} \mathrm{R}^{\mathrm{i}} w^{\prime}$, but this would lead to relate words that are already semantically distant.

We should note that each concept (synsets) of WordNet has a specificity that is estimated through its depth in the WordNet of the conceptual tree induced by the "is-a" relation.

First, for each document we extract its terms, we only deal with lemmatized noun groups. Next we project this noun on WordNet to extract concepts. Note that for polysemic terms (e.g. ,claim ${ }^{\text {ee }}$ has 6 senses), we use a disambiguation method [6] in order to select the appropriate sense of the term in the text. This procedure is very time consuming.

For each query, we have extracted WordNet concepts, no disambiguation method is used at this stage, because the senses of a concept depend from users. So we choose to represent the query by all concept senses of its terms.
In the second step we calculate the value of parameters for each concept of the query: centrality, specificity and frequency. To calculate the centrality we have used, in this preliminary experiment, all WordNet relations $\mathrm{S}, \mathrm{I}^{-1} \mathrm{I}^{-1}, \mathrm{P}, \mathrm{P}^{-1}$, $\mathrm{D}$, or R.

The specificity is estimated through the "depth" in WordNet in the conceptual tree induced by the "is-a" relation.

The Frequency estimates the number of occurrences of a concept in a document. Moreover, words in the same "synset" will be counted together.

As mentioned above, as a query concept could be represented by different concepts of WordNet, (we did not disambiguate them). We then selected for each query concept its "right" representative in wordnet. We selected the concept-sense which has the highest centrality.

\section{B. Impact of Centrality and Specificity}

Our goal here is to evaluate the impact of each of the new factors. We evaluated different configurations.

Table 1 lists the result of the ranking model by assigning different value for each of $\alpha, \beta, \gamma$. We only listed effective results. Each column represents a specific configuration. The first result we notice is that centrality seems to be more effective than specificity. The combination of the three parameters (avoiding zero for $\mathrm{C}$ and $\mathrm{F}$ ) columns 7 and 8 for both values of $\alpha=1$ or 2 gives the best results, with a slight benefit for $\alpha=2$. For the remaining experiments we consider the formula of column 8 only

\begin{tabular}{|c|c|c|c|c|c|c|c|}
\hline RSV & $\sum \mathrm{C}$ & $\sum \mathrm{S}$ & $\sum \mathrm{C}^{* \mathrm{~S}}$ & $\sum(1+\mathrm{C}) * \mathrm{~S}$ & $\begin{array}{c}\sum(1+\mathrm{C})^{*} \\
(1+\mathrm{F})\end{array}$ & $\begin{array}{c}\sum(1+\mathrm{C})^{*} \\
(1+\mathrm{F}) * \mathrm{~S}\end{array}$ & $\begin{array}{c}\sum(1+\mathrm{C})^{2 *}(1+ \\
\mathrm{F})^{*} \mathrm{~S}\end{array}$ \\
\hline P5 & 0,4833 & 0,3167 & 0,4875 & 0,4917 & 0,4917 & 0,5042 & 0,5 \\
\hline P10 & 0,4625 & 0,3312 & 0,4521 & 0,45 & 0,4917 & 0,4792 & 0,4917 \\
\hline P15 & 0,4444 & 0,3556 & 0,4417 & 0,4403 & 0,4667 & 0,4681 & 0,4694 \\
\hline P20 & 0,4344 & 0,3583 & 0,4281 & 0,4198 & 0,4469 & 0,4469 & 0,4562 \\
\hline P30 & 0,4174 & 0,3528 & 0,4118 & 0,4125 & 0,4257 & 0,4264 & 0,4375 \\
\hline P100 & 0,365 & 0,364 & 0,3648 & 0,3646 & 0,3642 & 0,3642 & 0,364 \\
\hline
\end{tabular}

Table 1: Impact of different combinations of factors

\section{Comparison of Obtained Results with BM25 Ranking Function}

In this step we compare our results to the baseline, these results are presented in Table 2 . It lists the precision at top-n document (top5, top10, top20, top30 and top 100) obtained for the 50 queries used for evaluating TREC1 collection.

In the obtained results, it is patent that the precision of our model is better, at almost all levels, than those of the baseline. The third column of Table 2, lists the improvement, we notice a clear improvement particularly at the top documents $(10,35 \%$ at top- 5 documents, $8,52 \%$ at top- 10 and $6,15 \%$ at top-15). 


\begin{tabular}{|c|c|c|c|}
\hline & $\mathbf{B M 2 5}$ & $\sum(\mathbf{1 + C})^{\mathbf{2 *}}(\mathbf{1 + F}) * \mathbf{S}$ & $\mathbf{( \% \mathbf { i m p } )}$ \\
\hline P5 & 0,4531 & 0,5 & $10,35 \%$ \\
\hline P10 & 0,4531 & 0,4917 & $8,52 \%$ \\
\hline P15 & 0,4422 & 0,4694 & $6,15 \%$ \\
\hline P20 & 0,4459 & 0,4562 & $2,31 \%$ \\
\hline P30 & 0,4299 & 0,4375 & $1,77 \%$ \\
\hline P100 & 0,3678 & 0,364 & $-1,03 \%$ \\
\hline
\end{tabular}

Table 2: Comparison of obtained results to the ones given by BM25

If we conceptually compare both approaches one could say that the difference (the benefit) could only come from the document and the query representation. In fact, BM25 used single words whereas in the proposed approach concepts are used. To alleviate this problem we compared our approach to a conceptual approach, based on WordNet, and proposed by Baziz [6].

\section{Comparison of Obtained Results with a Concept-Based Method}

In this step we compare our results to those obtained by a concept-based method proposed by Baziz [6]. In this concept-based approach, the documents and the queries are indexed using concept based detection. In the method each term of the document (query) is represented by a set of weighted concepts (ontology nodes). The concepts are detected in the same way used in our approach. The weight of a concept in a document is estimated using a "c_score". More precisely, each term $t_{l}$ of a document $d$ has $n$ senses where the $k$-th sense " $C_{k}^{i}$ " of $t_{l}$ corresponds to a concept in WordNet. To find which concepts to affect to the term $t_{l}$, the method affect a weight " $\mathrm{C}_{-}$score $\left(\mathrm{C}_{\mathrm{k}}^{\mathrm{i}}\right)$ " to each possible sense " $C_{k}^{\mathrm{i}}$ " using this function:

$$
C_{-} \text {score }\left(C_{k}^{i}\right)=\sum_{\substack{l \in[1 m m] l \neq i \\ j \in[1 m m]}} \mathbb{P}_{i, 1}\left(c_{k}^{i}, c_{j}^{1}\right)
$$

where $m$ represents the number of terms of document $d, n$ the number of senses of the term $t_{l}$ where each sense corresponds to a concept " $C_{j}^{l n}$ in WordNet and $P_{i, l}$ is a semantic measure of similarity between two concepts, in this case is the Resnik measure [8].

the $C_{-}$score for a term $T_{i}$ is represented by the best concept $\overline{C_{i}}$ that better represents the sense of the term $T_{i}$ and maximizes $\mathrm{C}$ _score:

$$
\mathrm{C}_{\mathrm{i}}=\mathrm{c}_{-} s \text { core }\left(\mathrm{T}_{\mathrm{i}}\right)=\operatorname{ArgMax}_{\mathrm{k}=1 \mathrm{n} n}\left\|\mathrm{C}_{-} \operatorname{score}\left(\mathrm{C}_{\mathrm{k}}^{\mathrm{i}}\right)\right\|
$$

And the ranking function that they use in their model is the following:

$$
\operatorname{RSV}(\mathrm{q}, \mathrm{d})=\sum_{\mathrm{t}=\mathrm{q}}\left(\mathrm{C}_{-} \text {score }(\mathrm{t})+\operatorname{Classic}(\mathrm{t})\right)
$$

where: Classic is a measure of BM25 TF.IDF formula.

\begin{tabular}{|c|c|c|c|}
\hline & Baziz & $\sum(\mathbf{1 + C})^{2 *}(\mathbf{1}+\mathbf{F}) * \mathbf{S}$ & $\mathbf{( \% i m p )}$ \\
\hline P5 & 0,32 & 0,4833 & $52,60 \%$ \\
\hline P10 & 0,3313 & 0,4917 & $48,42 \%$ \\
\hline P15 & 0,3417 & 0,4694 & $37,37 \%$ \\
\hline P20 & 0,3521 & 0,4552 & $29,28 \%$ \\
\hline P30 & 0,3542 & 0,4375 & $23,52 \%$ \\
\hline P100 & 0,3625 & 0,364 & $0,41 \%$ \\
\hline
\end{tabular}

Table 3: Comparison of obtained results with a concept based indexing method

According the results listed in Table 3, we clearly notice that the precision of our model is largely better, at all levels, than those of the conceptual model of Baziz. The third column presents the improvements.

One of the interesting results one may draw from these experiments concerns the concept weighting. It is well known in IR that factors such as tf, idf and document length are effective for IR approaches based on single terms. These factors are not effective for measuring the significance of concepts (or phrases). The proposed factors could be considered as new sources of evidence that could be added to any conceptual weighting schema as those proposed by Baziz [6], Robertson [2], Salton [29], Jin [31] and croft [11]

\section{CONCLUSiOn AND PeRspectives}

We have proposed a new document ranking method given a query. The method that we have described is based on semantic representation of the contents of texts. In particular, we have advocated the use of specificity and centrality evaluations in order to weight a document facing a query. These criteria aggregated with the traditional $t f$ provide a good ranking function. The idea of specificity and centrality has been already proposed in [27][37] and was tested in ,summarization ${ }^{\text {ee }}$ of texts, without testing the influence of these criteria in the information retrieval domain. The proposed aggregation function of the centrality, specificity and frequency criteria gives good results; other aggregations should be tested for trying to enhance the obtained results.

We have illustrated the procedure for reordering results of the Okapi function. But the same procedure could be applied in order to extract pertinent documents from all the document collection in response to a query.

We may also think of testing normalized versions of centrality, specificity and term frequency in order to improve our results.

While the used parameters (Centrality, Specificity and Frequency) give good results, we may also test the influence of other parameters such as the "c_score" used by Baziz [6] to estimate the similarity of a concept in a document in combination with other parameters. Another line of research will be introduction of the Okapi function in combination with the other parameters. 


\section{ACKNOWLEDGMENT}

This work was in part supported by OSEO under the QUAERO project.

\section{REFERENCES}

[1] G. Salton (1989). Automatic Text Processing. Addison-Wesley .

[2] S. E. Robertson, C. J. van Rijsbergen, and M. F. Porter (1981). Probabilistic models of indexing and searching. R.N. Oddy, S.E. Robertson, C.J. van Rijsbergen, and P.W. Williams, editors, Information Retrieval Research, (pp. 35-56). London: Butterworths.

[3] H.R. Turtle, W. C. Croft (1991). Evaluation of an inference networkbased retrieval model. ACM Transaction on Information Systems 9 (3) , 7187-7222.

[4] K. Sparck Jones, S.E. Robertson, D. Hiemstra, and H. Zaragoza (2003). Language modelling and relevance. In Croft W.B. and Lafferty J., editors, Language Modelling for Information Retrieval (pp. 57-70). Kluwer Academic Publishers.

[5] S. Robertson, S. Walker (1994). Some simple effective approximations to the 2-poisson model for probabilistic weighted retrieval. in: Proceedings of the International ACM-SIGIR Conference, (pp. 232-241).

[6] M. Baziz, M. Boughanem, G. Pasi, H. Prade (2006). A fuzzy logic approach to information retrieval using an ontology-based representation of documents. In: Fuzzy Logic and the Semantic Web. (E. Sanchez, Ed.), Elsevier, 363-377.

[7] G. Salton, M. McGill (1987). Introduction to Modern Information Retrieval. McGraw-Hill.

[8] H. P. Luhn (1957). A Statistical Approach to Mechanized Encoding and Searching of Literary Information. IBM J. Research and Development, 1 (4), 309-317.

[9] C.S. Yang, G. Salton (1973). On the Specification of Term Values in Automatic Indexing. J. Documentation, 351-372.

[10] K. Sparck-Jones (1973). Index Term Weighting, Information storage and retrieval. Information Storage and Retrieval, 9 (11), 619-633.

[11] R. Krovetz, B. Croft (1992). Lexical ambiguity and information retrieval. ACM Trans. on Information Systems, 10 (2), 115-141.

[12] M. Sanderson (1994). Word sense disambiguation and information retrieval. Proc. of ACM SIGIR'94 Conf., 17, 142-151.

[13] D. Yarowsky (1995). Unsupervised word sense disambiguation rivaling supervised methods. Proc. 33rd Annual Meeting Assoc. for Computat. (pp. 189-196). Linguistics, Cambridge, Ma.

[14] S. Deerwester, S. T. Dumais, T. K. Landauer, G. W. Furnas, R. A Harshman (1990). Indexing by latent semantic analysis. J. of the Society for Information Science, 41 (6) , 391-407.

[15] M M. Montes-y-Gómez, A. López-López, A. Gelbukh (2000). Information retrieval with conceptual graph matching. Proc. DEXA'00 (pp. 312-321). Greenwich: Springer, LNCS 1873.

[16] R. Thomopoulos, P. Buch, O. Haemmerlé (2003). Representation of weakly structured imprecise data for fuzzy requiting. Fuzzy Sets and Systems, 140, 111-128.

[17] J. Gonzalo, F. Verdejo, I. Chugur, J. Cigarrán (1998). Indexing with WordNet synsets can improve text retrieval. Proc.COLING/ACL'98 Workshop on Usage of WordNet for Natural Language Processing. http://vldbarc.org/dblp/db/jou rnals/corr/corr9808.html\#cmp.

[18] R. Richardson, A. Smeaton, J. Murphy (1994). Using WordNet as a knowledge base for measuring semantic similarity between words. Proc. of AICS Conf. (http://citeseer.ist.psu.edu/187048.html). Trinity College, Dublin: Trinity College.

[19] D. Widyantoro, J. Yen (2001). A fuzzy ontology-based abstract search engine and its user studies. Proc.10th IEEE Inter. Conf. on Fuzzy Systems, (pp. 1291-1294). Melbourne, Australia.
[20] J.A. Olivas, P.J. Garcés, F.P. Romero (2003). An application of the FIS-CRM model to the FISS metasearcher: Using fuzzy synonymy and fuzzy generality for representing concepts in documents. Int. J. of Approximate Reasoning, 34, 201-219.

[21] M. Boughanem, G. Pasi, H. Prade (2004). A fuzzy set approach to concept-based information retrieval. Proc. 10th Inter. Conf. on Information Proces. and Mgmt. of Uncertainty in Knowl-Based Syst.(IPMU'04), (pp. 1775-1782). Perugia, July 4-9.

[22] G. Boone (1998). Concept features in Re:agent. An intelligent email agent. Proc. $2^{\text {nd }}$ Conf. on Autonomous Agents, (pp. 141 - 148). May 10-13, Minneapolos/St Paul.

[23] G. A. Miller (2009). WordNet - About Us. WordNet. Princeton University.. "http://wordnet.princeton.edu"

[24] M. Boughanem, K. Sauvagnat, C. Laffaire (2003). Mercure at TREC'2003, Web track - Topic Distillation Task. Text REtrieval Conference (TREC 2003). Gaithersburg, Maryland, USA, 18-21 /11/2003, National Institute of Standards and Technology (NIST).

[25] M. Porter (1980). An algorithm for suffix stripping. Program, 14 (3), 130-137.

[26] G. Salton and M.J. McGill (1984). Introduction to Modern Information Retrieval. McGraw-Hill Int. Book Co .

[27] M. Boughanem, H. Prade, O. Bouidghaghen (2008). Extracting topics in texts : Towards a fuzzy logic approach. Proc. 12th International Conference on Information Processing and Management of Uncertainty in Knowledge-based Systems (IPMU 2008) (pp. 17331740). Málaga, Spain, 22-27-June, L. Magdelena, M. Ojeda-Aciego, J. L. Verdegay (Eds.)

[28] P. Resnik (1999). Semantic Similarity in a Taxonomy: An Information-Based Measure and its Application to Problems of Ambiguity in Natural Language. Journal of Artificial Intelligence Research (JAIR), 11, 95-130.

[29] G. Salton, C. Buckley (1988). Term-weighting approaches in automatic text retrieval. Information Processing and Management: an International Journal 24 (5), 513-523.

[30] S. E. Robertson, S. Walker, M. M. HancockBeaulieu, M. Gatford and A. Payne (1996). Okapi at TREC-4. The Fourth Text REtrieval Conference (TREC-4).

[31] J. Rong, Y. Joyce, L. Si Chai (2005). Learn to weight terms in information retrieval using category information. ACM International Conference Proceeding Series; Vol. 119, Proceedings of the 22nd International Conference on Machine Learning (pp. 353 - 360). Bonn, Germany : ACM, New York, NY, USA.

[32] Y.-P. Huang, L. J. Kao, T. Tsai, and D. Liu (2003). Using Fuzzy Centrality and Intensity Concepts to Construct an Information Retrieval Model. Proc. IEEE International Conference on Systems, Man and Cybernetics, 5-8 Oct.

[33] P. Mutschke (2001). Enhancing Information Retrieval in Federated Bibliographic Data Sources Using Author Network Based Stratagems. In: Research and Advanced Technology for Digital Libraries, LNCS 2163, Springer Verlag, pp 287-299.

[34] H. Zhuge, J. Zhang. Topological Centrality and Its Applications. arXiv:0902.1911v1 [cs.IR] 11 Feb 2009.

[35] G. Erkan, D. R. Radev (2004). LexRank: Graph-based Lexical Centrality as Salience in Text Summarization. Journal of Artificial Intelligence Research 22, pp 457-479.

[36] J. M. Kleinberg, R. Kumar, P. Raghavan, S. Rajagopalan and A. S. Tomkins (1999). The Web as a Graph: Measurements, Models, and Methods. LNCS 1627, Springer Verlage. pp. 1-17.

[37] O. Bouidghaghen, M. Boughanem, H. Prade, I. Mallak (2009). A fuzzy logic approach to topic extraction in texts. International Journal of Uncertainty, Fuzziness and Knowledge-Based Systems, 17, N. Suppl. 1, 81-112. 